\title{
Effect of motility on the transport of bacteria populations through a porous medium
}

\author{
Adama Creppy* \\ Laboratoire FAST, Univ. Paris-Sud, CNRS, Université Paris-Saclay, F-91405, Orsay, France
}

Eric Clément ${ }^{\dagger}$

Physique et Mécanique des Milieux Hétérogenes (UMR 7636 ESPCI/CNRS/Univ. P.M. Curie/Univ. Paris-Diderot), 10 rue Vauquelin, 75005 Paris, France

Carine Douarche $e^{\ddagger}$

Laboratoire FAST, Univ. Paris-Sud, CNRS, Université Paris-Saclay, F-91405, Orsay, France

\author{
Maria Veronica D’Angelo ${ }^{\S}$ \\ Universidad de Buenos-Aires, Facultad de Ingeniería, GMP-LIA-FMF, CONICET, \\ Paseo Colón 850, 1063 Buenos Aires, Argentina
}

Harold Auradoull

Laboratoire FAST, Univ. Paris-Sud, CNRS, Université Paris-Saclay, F-91405, Orsay, France.

(Received 6 February 2018; published 16 January 2019)

\begin{abstract}
The role of activity on the hydrodynamic dispersion of bacteria in a model porous medium is studied by tracking thousands of bacteria in a microfluidic chip containing randomly placed pillars. We first evaluate the spreading dynamics of two populations of motile and nonmotile bacteria injected at different flow rates. In both cases, we observe that the mean and the variance of the distances covered by the bacteria vary linearly with time and flow velocity, a result qualitatively consistent with the standard geometric dispersion picture. However, quantitatively, the motile bacteria display a systematic retardation effect when compared to the nonmotile ones. Furthermore, the shape of the traveled distance distribution in the flow direction differs significantly for both the motile and the nonmotile strains, hence probing a markedly different exploration process. For the nonmotile bacteria, the distribution is Gaussian, whereas for the motile ones, the distribution displays a positive skewness and spreads exponentially downstream akin to a $\Gamma$ distribution. The detailed microscopic study of the trajectories reveals two salient effects characterizing the exploration process of motile bacteria: (1) the emergence of an "active" retention effect due to an extended exploration of the pore surfaces and (2) an enhanced spreading at the forefront due to the transport of bacteria along "fast tracks" where they acquire a velocity larger than the local flow velocity. We finally discuss the practical applications of these
\end{abstract}

\footnotetext{
*adama.creppy@u-psud.fr

†eric.clement@upmc.fr

†carine.douarche@u-psud.fr

§veronica.dangelo@gmail.com

"auradou@fast.u-psud.fr
} 
effects on the large-scale macroscopic transfer and contamination processes caused by microbes in natural environments.

DOI: 10.1103/PhysRevFluids.4.013102

\section{INTRODUCTION}

Understanding the transport of microorganisms in heterogeneous media is a question dealing with a large variety of scientific and technological domains such as bacteriology, ecology, sciences for environment, petroleum research, or medicine. For example, bacteria are now used as vectors for fighting cancers [1,2]. The oil industry also considers the potential of bacteria injection to enhance oil recovery [3]. Nowadays, bioremediation techniques are developed in which contaminants trapped in the ground are targeted and then decomposed or fixed by bacteria [4]. Some biotechnologies require the isolation of specific microbial strains, and these processes need adapted filtration or sorting techniques [5].

A better understanding of how microorganisms are transported trapped or dispersed in disordered porous media is not only a key to the development of future innovative applications but also may shed new lights on the strategies that microorganisms use to maximize their survival and proliferation abilities in natural conditions.

In the past decades, modeling the transport of microorganisms was essentially driven by an increasing concern about pollution of ground waters [6,7]. The approaches used to model the transport in porous media are currently based on the standard advection-dispersion equation including biological processes such as growth and mass exchange with the grains, through phenomenological coefficients derived from breakthrough curves [8-13]. The focus has been mostly put on the adhesion properties influenced by chemical processes like $\mathrm{pH}$ or ionic strength. The final outcome of these analyzes is often disappointing as most of the reports conclude on difficulties to scale up laboratory column experiments $[14,15]$. Surprisingly, little is known about the influence of microbial motility on the retention processes inside the pores or the way the swimming ability contributes to the transport in confined channels, although some studies bring the evidence of an undeniable influence [16,17]. It is only recently that a macrocontinuum transport model based on a generalized Taylor-dispersion approach was undertaken to study the dispersion in model porous media made of a periodic array of pillars [18].

Recent developments in microfluidic techniques provide a new and efficient tool that can be used to visualize transport processes of bacteria and allow to assess the influence of well-controlled environments like the dynamical trapping caused by retention by obstacle [19,20]. For example, Ford and co-workers [21] quantified the enhancement of transverse migration of bacteria due to chemotaxis. Additionally, other developments in microfluidic technology provided a way to observe the movement of bacteria in "simple" flows and to study the rheotactic coupling between bacteria motility and flows in channels [22] as well as the coupling of the shear rate with flagellar helicity [23]. Furthermore, many studies demonstrate the specificity and variety of motions of motile microorganisms in confined geometries such as a circular motion at planar surfaces [24], an upstream swimming in response to shear at surfaces [23,25,26], in capillary tubes [27], or at edges [28], as well as a transverse drift at surfaces [26,28] also due the coupling with flagellar helicity. This has important macroscopic consequences on the organization of bacteria in a flow, such as the depletion of bacteria at zero-shear regions [22] or the accumulation of swimmers at surfaces [29], at stagnation points [30], or downstream a channel constrictions [31].

It is then clear from all these examples that bacteria spend a significant fraction of time on surfaces which can be seen as inducing a dynamical trapping process essentially due to hydrodynamic interactions. However, what still needs to be understood is the contribution of these processes to the transport at a macroscopic scale.

Our study demonstrates how physical processes associated with the interplay between motility and flow in a disordered porous medium lead to significant effects on the macroscopic transport 
(a)

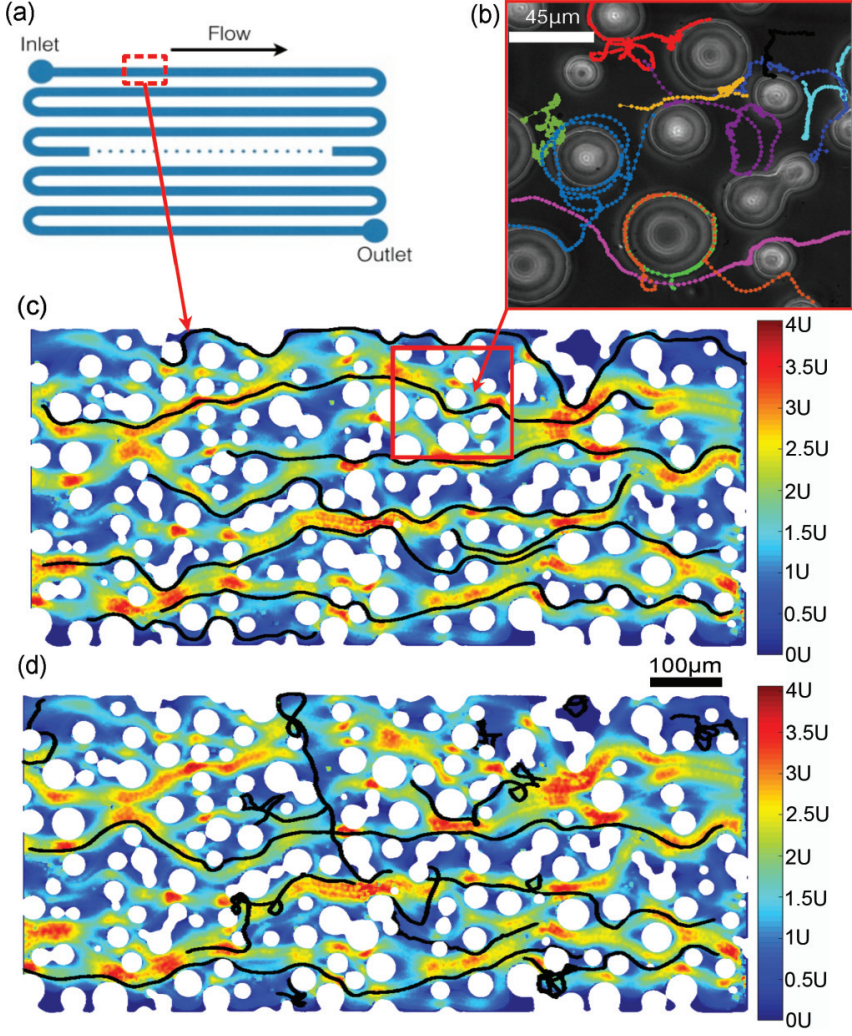

FIG. 1. (a) Schematic view of the overall microfluidic chip. (b) Colored lines representing trajectories of motile bacteria in the porous space. (c, d) Superimpositions of the velocity field obtained with passive tracers and $E$. coli trajectories (black solid lines) for nonmotile (c) and motile (d) bacteria during $\Delta t=10 \mathrm{~s}$ at a flow rate $U=36 \mu \mathrm{m} / \mathrm{s}$. White circles represent the pillars (average diameter $d=35 \mu \mathrm{m}$ ). Images are recorded with a $10 \times$ objective. The field of view is $1 \mathrm{~mm} \times 0.5 \mathrm{~mm}$.

properties. To this purpose, we designed a microfluidic environment in which motility and pore geometry are the dominant ingredients influencing the hydrodynamic dispersion of a bacterial fluid. The microfluidic channel includes some of the random structural heterogeneities of natural pore structures. We also used motile and nonmotile bacteria that do not stick to the surfaces. This choice of strains with different motility is important to identify the specificity of motility-borne effects, as was already noticed in studies of bacterial swarming [32,33]. The study was performed in a large window of flow rates, and in this report we provide crucial experimental observations to help develop new physical transport models.

\section{MATERIALS AND METHODS}

\section{A. Microfluidic chip fabrication}

The microfluidic chip is designed by standard photolithography techniques. The channel geometry is a serpentine of rectangular section (width $=500 \mu \mathrm{m}$, height $=100 \mu \mathrm{m}$ ) filled with circular pillars [see Fig. 1(b)]. The pillar centers are randomly distributed along the channel, and their diameter are randomly chosen among four values $(20,30,40$, and $50 \mu \mathrm{m})$. Channels are designed to be filled at $33 \%$. The average diameter of the obstacles is thus $d=35 \mu \mathrm{m}$, the average distance between two close neighbors is $\sim 10 \mu \mathrm{m}$. Channels are made of polydimethyle siloxane (PDMS), and the chip is bonded onto a glass plate $(5.5 \mathrm{~cm} \times 5.5 \mathrm{~cm})$ using a plasma cleaner. 
PMDS is permeable to oxygen allowing a continuous oxygenation of the suspension ensuring the constant swimming activity of the bacteria during the experiments [34].

\section{B. Strain and culture}

A fluorescent Escherichia coli RP437 strain is used. The fluorescence is obtained by transforming the wild-type E. coli RP437 with a plasmid coding for a yellow fluorescent protein. The bacteria are cultured overnight at $30^{\circ} \mathrm{C}$ and shaken at $240 \mathrm{rpm}$ in $\mathrm{M} 9$ minimal medium supplemented with $1 \mathrm{mg} / \mathrm{ml}$ casamino acids, $4 \mathrm{mg} / \mathrm{ml}$ glucose, and $25 \mu \mathrm{g} / \mathrm{ml}$ chloramphenicol at $0.05 \%$. The growth medium is then removed by centrifugation $(2300 \mathrm{~g}$ for $10 \mathrm{~min})$. The bacterial suspension is then rinsed with milliQ water to remove any residuals from the overnight culture. The bacterial population is finally resuspended into a motility buffer containing $10 \mathrm{mM}$ phosphate buffer $(\mathrm{pH}=7), 0.1 \mathrm{mM}$ EDTA, $1 \mu \mathrm{M}$ L-methionine, and $10 \mathrm{mM}$ sodium L-lactate. To avoid bacterial sedimentation, the suspension is mixed with Percoll $(1 \mathrm{v} / \mathrm{v})$. In this suspending medium, bacteria are able to live and swim but do not divide. The optical density (OD) is measured at $600 \mathrm{~nm}$ using a spectrophotometer BEL SP 1105 in order to determine the bacterial concentration. All experiments are performed at a low cell concentration (OD $\sim 2.5 \times 10^{-3} \sim 3$ bacteria/ $\mu$ l) but high enough to get good statistics for tracking measurements. Under those conditions, the average swimming velocity is measured to be $\overline{v_{b}}=19 \pm 4 \mu \mathrm{m} / \mathrm{s}$ and remains roughly constant for several hours. Nonmotile bacteria are prepared by keeping the suspension at $4{ }^{\circ} \mathrm{C}$ for $5 \mathrm{~h}$. The suspension is then maintained at $22^{\circ} \mathrm{C}$ for $30 \mathrm{~min}$ before its use. No sign of motility is detected over the entire duration of the experiments (i.e., $4 \mathrm{~h}$ ).

\section{Experimental protocol}

Observations are performed with a camera mounted on a Leica DMI 6000 inverted microscope driven by $\mu$ Manager software [35]. Two cameras are used: (1) a IDS CMOS camera for phasecontrast visualization and (2) a Hamamatsu ORCA-Flash 4.0 camera when the fluorescence mode is preferred. Most of the movies are recorded at different places located at about $2 \mathrm{~cm}$ from the inlet of the channel, which corresponds to a distance of approximately 20000 cell bodies or 600 grain sizes. First, the microfluidic device is filled with motility buffer and Percoll (50\%). A $100 \mu 1$ syringe is filled with the bacterial suspension and loaded on a computer-controlled Nemesys pump. Most of the experiments are done with a $10 \times$ objective, the field of depth of which is $\sim 30 \mu \mathrm{m}$ (about $1 / 3$ of the channel height). Before the image acquisition, the objective is moved vertically to focus on the middle of the channel. The images of Fig. 1(b) and the movie are obtained using a $63 \times$ objective (field of depth $1 \mu \mathrm{m}$ ). Sequences of 3000 images are recorded with a frame rate that depends on the flow velocity. It is chosen such that the average distance traveled by the bacteria between two successive frames is $\sim 5 \mu \mathrm{m}$. Before the acquisition of the image sequence, three to five short sequences of 100 images are taken every $10 \mathrm{~min}$ and processed systematically to verify the steadiness of the flow.

\section{Image analysis and bacteria tracking}

Bacteria tracking is done using the "trackmate" plug-in of the image analysis freeware Fiji [36]. Prior to the analysis, the average image obtained over the whole sequence lasting between 32 and $450 \mathrm{~s}$ is subtracted from each image. The tracking procedure has two steps: (1) bacteria detection and (2) frame by frame linking of positions to build the individual tracks. The output is a data file giving for each detected bacterium its position $x \vec{e}_{x}+y \vec{e}_{y}$ as a function of time $t$. Figures 1(c) and 1(d) show examples of trajectories obtained with this method. Thousands of trajectories are obtained at the same field of view along the channel for motile and nonmotile bacteria but also for latex beads of diameter $2 \mu \mathrm{m}$. The flow velocity field $\vec{v}(x, y)$ is derived from these passive tracer trajectories. The coarse-grained spatial resolution used to determine the flow field corresponds to a square of $5 \mathrm{px} \times 5 \mathrm{px}(2.75 \mu \mathrm{m} \times 2.75 \mu \mathrm{m})$. Figures $1(\mathrm{c})$ and $1(\mathrm{~d})$ show a typical velocity field 
(a)

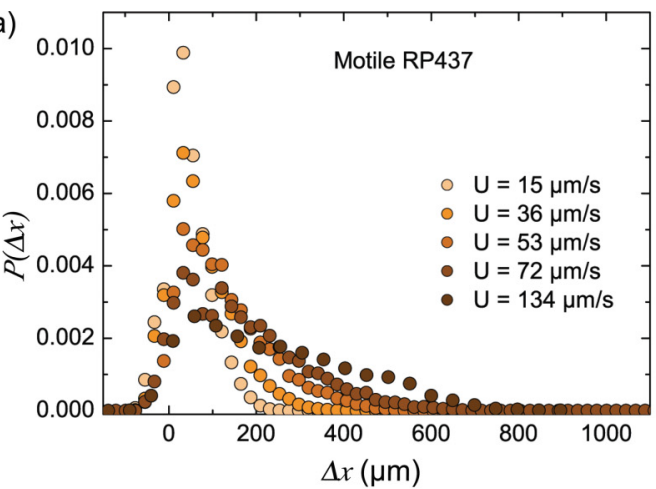

(c)

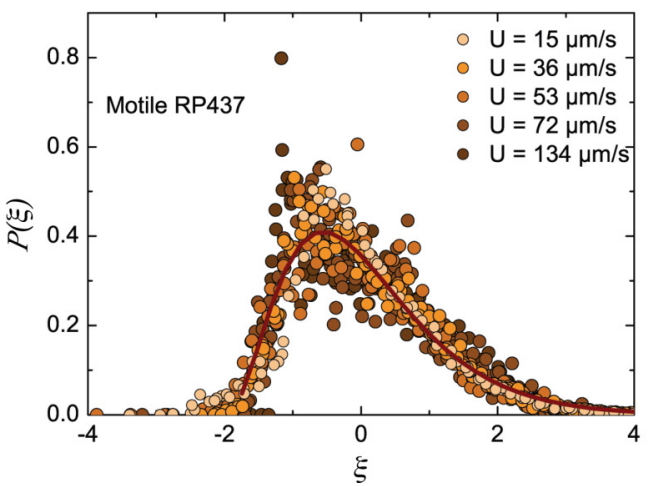

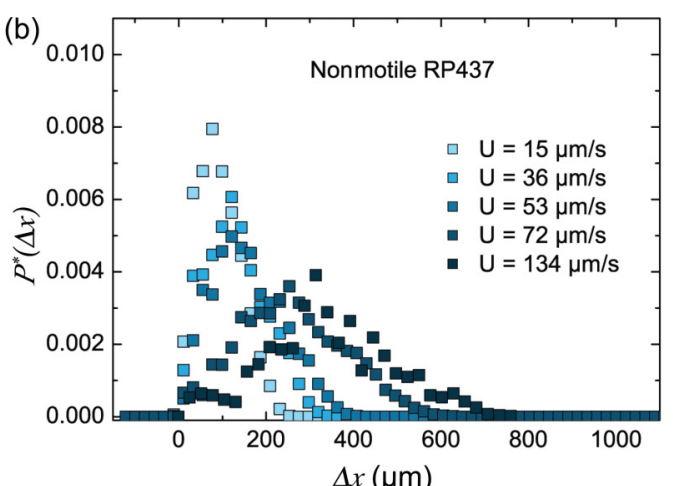

(d)

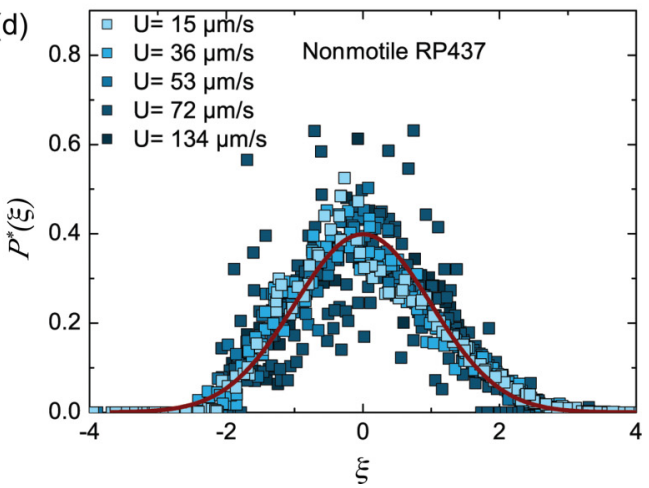

FIG. 2. (a, c) Distributions $P(\Delta x, \Delta t)$ of the distances $\Delta x$, traveled along the flow direction at a time $\Delta t=3 \mathrm{~s}$, for motile (a) and nonmotile (c) bacteria at different flow velocities. (b, d) Normalized distributions $P^{*}(\xi)$ where $\xi=\frac{\Delta x-\overline{\Delta x}}{\sigma_{x}}$ for the motile (b) and the nonmotile (d) bacteria. For each flow velocity, five different $\Delta t$ are represented ( $\Delta t=1,3,5,7$, and $9 \mathrm{~s}$ ). Solid red line: (b) a Gaussian distribution of zero mean and unit variance and (d) a best fit from a $\Gamma$ distribution.

obtained using this method and superimposed with a few trajectories of nonmotile [Fig. 1(c)] and motile [Fig. 1(d)] bacteria. The conversion between the flow rate $Q$ imposed by the pump and the average flow velocity $U$ is obtained from a series of calibration experiments performed with the passive tracers.

\section{RESULTS}

Differences between motile and nonmotile bacteria are qualitatively illustrated in the trajectories displayed in Figs. 1(c) and 1(d). While the trajectories of nonmotile bacteria are primarily oriented along the flow direction and follow the streamlines [Fig. 1(c)], the motile bacteria display more erratic trajectories with a significant deviation from the flow lines [Fig. 1(d)]. Magnification at the level of the obstacles [Fig. 1(b)] reveals that the motile bacteria trajectories are interspersed with moments in which the bacteria change direction, move upstream, and travel back and forth from the vicinity of one obstacle to another, much like a ball in a pinball game.

Quantitatively, we characterize the transport and dispersion properties by computing the distributions of distances traveled by the bacteria along both the flow direction $(\Delta x)$ and the transverse direction $(\Delta y)$ over a time interval $\Delta t$. Figure 2 shows the distributions $P(\Delta x, \Delta t)$ for a fixed time interval $\Delta t=3 \mathrm{~s}$ for motile [Fig. 2(a)] and nonmotile [Fig. 2(b)] bacteria, at different flow rates. Significant differences in the spreading dynamics and in the shape are immediately visible. In the following, we provide an account for the transport of motile and nonmotile bacteria by a 
quantitative analysis of these distributions. We define the averages of the displacement distributions over the trajectories $(\overline{\Delta x}$ and $\overline{\Delta y})$ as well as the standard deviations $\left(\sigma_{x}\right.$ and $\left.\sigma_{y}\right)$ along the $x$ and $y$ axis, respectively. We also determine the distributions of the adimensionalized variables: $\xi=\frac{\Delta x-\overline{\Delta x}}{\sigma_{x}}$ and the skewness of the distribution along the flow $S k_{x}$.

\section{A. Dispersion processes}

To highlight the differences between the motile and the nonmotile bacteria, we characterize the shapes of the distributions of the adimensionalized transport distances along the flow $P^{*}(\xi)$ for the motile [see Fig. 2(c)] and for the nonmotile [see Fig. 2(d)] bacteria.

Importantly these distributions collapse on the same curve for all flow velocities $U$ and for time intervals corresponding to a mean displacement larger than one and up to six pore sizes $(\sim 210 \mu \mathrm{m})$ as long as the measurement can be performed. Then it shows that above the pore distance, one gets a converged stochastic process characterizing the transport and the dispersion of bacteria in the porous medium. For the nonmotile bacteria, the distribution is very close to a normal distribution (a Gaussian of zero mean, unit variance, and zero skewness). The correlation coefficient for the fit is 0.966 . This result is the one expected for classical geometrical dispersion of inert species in porous flows [37,38]. For motile bacteria, however, the rescaled distribution displays a significant mean skewness $\left(S k_{x} \sim 0.85\right)$ characterizing both a forward spreading and a retardation effect that can be seen on the maximum position (the mode) standing at a negative value $(\sim-0.5)$. For $\xi_{s}>-2$, the distribution can be approximately fitted using a $\Gamma$ distribution, which highlights the exponential decay of the forefront: $P^{*}(\xi)=\Gamma(k)^{-1} \xi_{0}^{-k}\left(\xi-\xi_{s}\right)^{k-1} \exp \left[-\left(\xi-\xi_{s}\right) / \xi_{0}\right]$ with $k \sim 3.366, \xi_{s}=-2$, and $\xi_{0} \sim 0.612$. Therefore at the front, the rescaled distribution decays more slowly for motile than for nonmotile bacteria, which is the sign of an enhanced transport process over a mesoscopic scale larger than the pore size.

\section{B. Average transport properties}

We study the influence of the flow velocity on the average position of the bacterial population. For all flow rates and for different time intervals $\Delta t$, the mean displacements $\overline{\Delta x}$ are computed. Figure 3(a) displays the average displacements as a function of the average distance $U \Delta t$ traveled by the fluid during the same time. We see that all data obtained for the nonmotile bacteria [square symbols in Fig. 3(a)] roughly collapse onto a line of slope $\sim 1$. This result indicates that the nonmotile bacteria progress in the porous medium with the average velocity of the fluid as would do passive tracers. However, for motile bacteria, we identify significant differences [circle symbols in Fig. 3(a)]. For a given mean flow velocity $U$, the average distance $\Delta x$ also increases linearly with time but with a slope smaller than 1 . This slope depends on the mean flow rate $U$, hence probing a retardation effect due to motility. A linear fit of the data gives an estimate of the average transport velocity $U_{M}$ of the motile bacteria. In Fig. 3(b) $U_{M}$ is plotted as a function of $U$, and we see for $U>50 \mu \mathrm{m} / \mathrm{s}$ an offset of the order of $20 \pm 12 \mu \mathrm{m} / \mathrm{s}$.

\section{Dispersion dynamics}

To quantify the spreading of the bacteria, we display the quadratic displacement of the longitudinal distance traveled by the bacteria rescaled by the mean size of the obstacles $\frac{\sigma_{x}^{2}}{d^{2}}$ as a function of the rescaled time $t^{*}=\frac{U \Delta t}{d}$ (see Fig. 4) for different average flow velocities. The data obtained for the motile [Fig. 4(a)] and the nonmotile [Fig. 4(b)] bacteria show essentially the same behavior. At short periods of time, a ballistic regime where $\sigma_{x}^{2}$ varies quadratically with time and at long periods (see insets in Fig. 4), a transition towards a diffusive regime for which $\sigma_{x}^{2} \sim t^{*}$. To extract the effective longitudinal diffusion coefficient, we use the standard Fürth function, $f\left(t^{*}\right)=\alpha\left[\beta t^{*}-\left(1-e^{-\beta t^{*}}\right)\right]$, derived from an Ornstein-Uhlenbeck process $[39,40]$ using $\alpha$ and $\beta$ as fitting parameters. The solid lines in Figs. 4(a) and 4(b) show the adjustments by a 

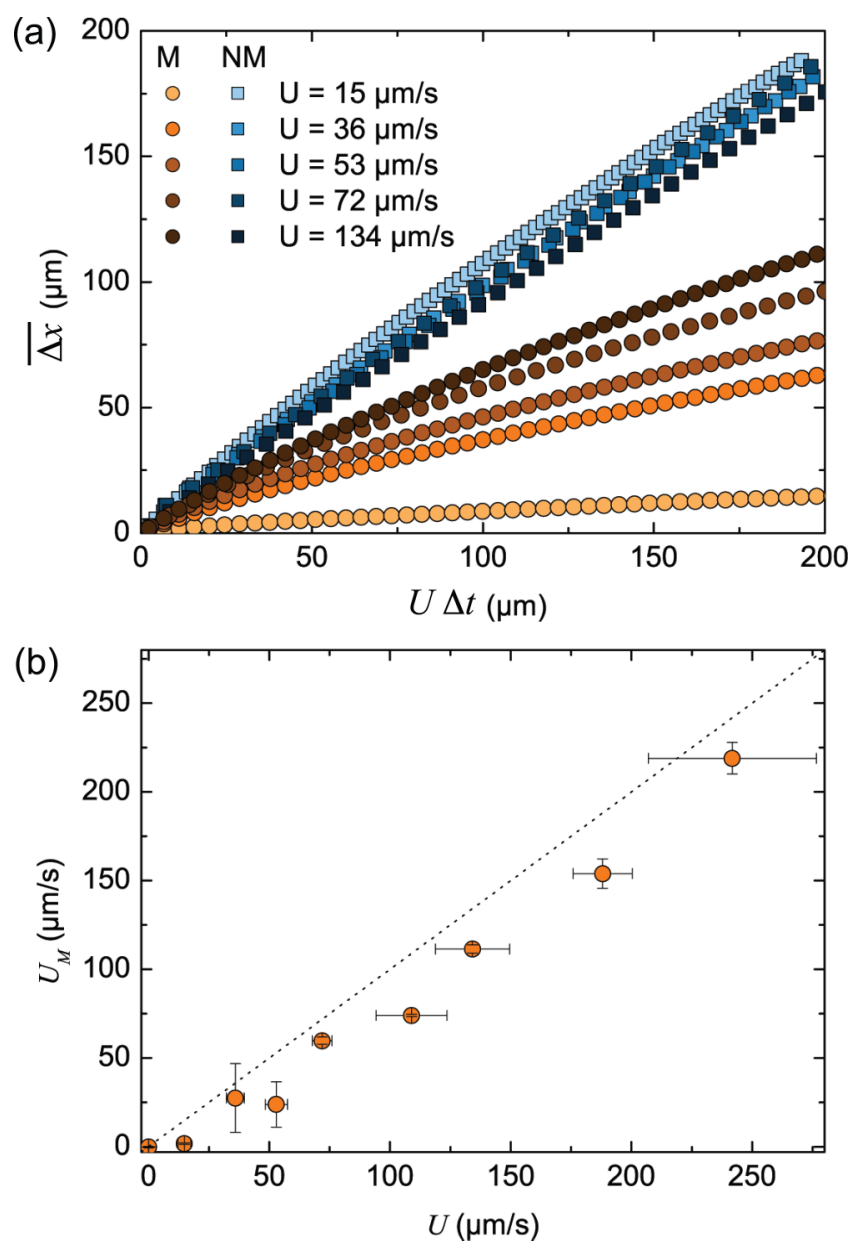

FIG. 3. (a) Mean positions of the bacterial population $\overline{\Delta x}$, as a function of the mean distance traveled by the fluid $U \Delta t$, for both motile (M) and nonmotile (NM) bacteria and for different flow rates. (b) Mean transport velocity of the motile bacteria $U_{M}$ as a function of the mean flow velocity $U$. The dotted line represents the $y=x$ function.

least-squares regression of our data. The adjustment is very good for all the flow velocities used and up to $t^{*} \sim 6$ pore sizes. The fitting parameters are then used to determine the dispersion coefficients $D_{L}=\frac{\alpha \beta d U}{2}$. The confidence intervals of $\alpha$ and $\beta$ were used to calculate the error bars of the dispersion coefficients. The crossover times correspond in all cases to a traveling distance comparable to a pore size.

The dispersion coefficients for nonmotile bacteria $\left(D_{L}^{N M}\right)$ and motile bacteria $\left(D_{L}^{M}\right)$ are displayed in Fig. 5 as a function of the mean flow velocity $U$. For nonmotile bacteria the dispersion coefficient is independent of the flow rate, and we extract a dispersivity length scale $\zeta^{N M}$ such that $D_{L}^{N M}=$ $\zeta_{L}^{N M} U$. One finds $\zeta_{L}^{N M}=50( \pm 16) \mu \mathrm{m}$, on the order of the pore size, which is a standard feature of geometrical dispersions occurring in disordered porous media [10,13].

For motile bacteria, we extract an effective dispersivity length scale $\zeta^{M}$ using the relation $D_{L}^{M}=$ $D_{0}^{M}+\zeta_{L}^{M} U$ where $D_{0}^{M}=210 \mu \mathrm{m}^{2} / \mathrm{s}$ is the zero-flow diffusivity extracted from the experiments. Note this value is three orders of magnitude larger than its equivalent for nonmotile bacteria. Therefore in the experimental uncertainties, the value of $\zeta^{M}$ seems to be equivalent to $\zeta^{N M}$ [see inset of Fig. 5(a)]. 

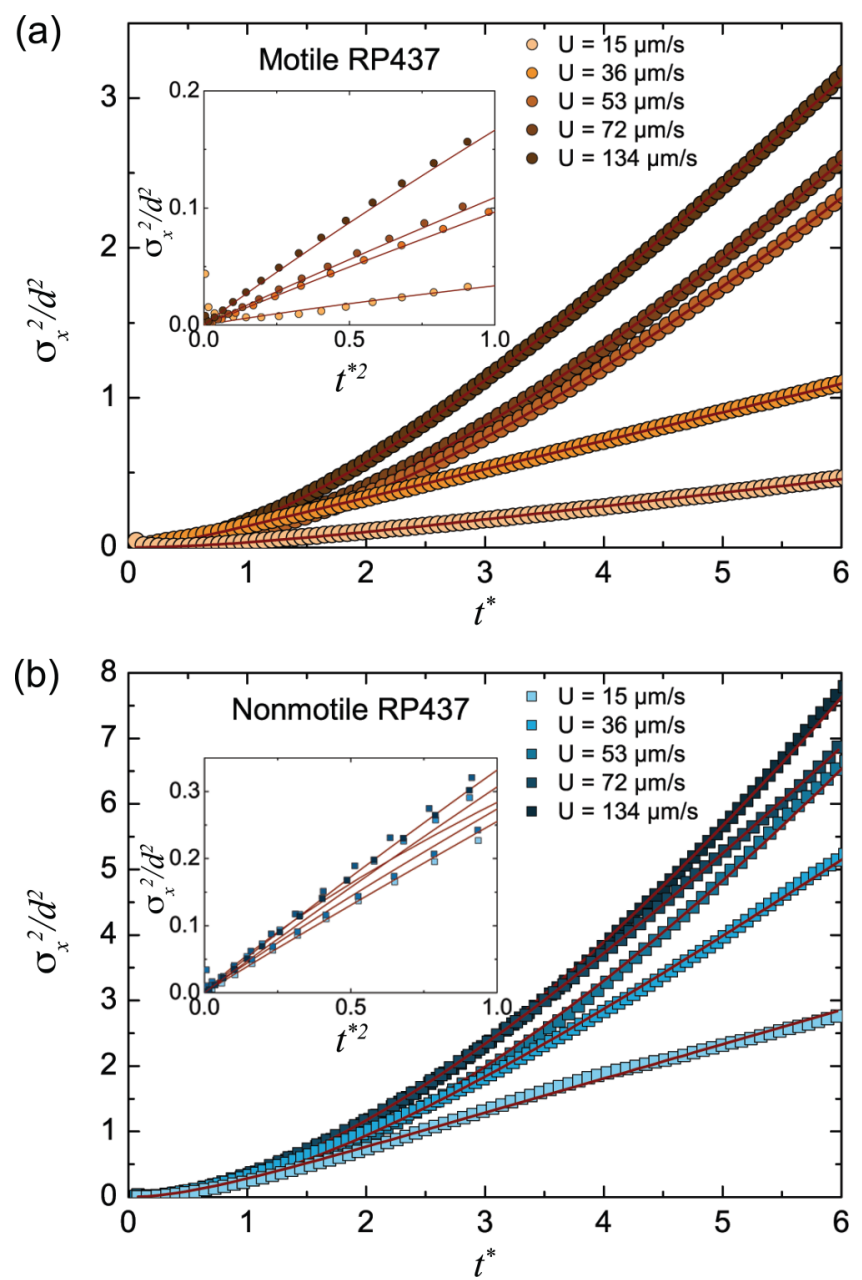

FIG. 4. (a, b) Variation of the dimensionless quadratic displacement $\frac{\sigma_{x}^{2}}{d^{2}}$ as a function of the normalized time $t^{*}=\frac{U \Delta t}{d}$ for different average flow velocities respectively for motile (a) and nonmotile (b) E. coli. Solid lines: adjustments by the function $f\left(t^{*}\right)=\alpha\left[\beta t^{*}-\left(1-e^{-\beta t^{*}}\right)\right]$. Insets represent the dimensionless quadratic displacement as a function of $t^{* 2}$ for less than one pore size.

We also find that bacteria motility induces a significant enhancement of the transverse exploration process. From a similar analysis as for longitudinal dispersion, we extract a transverse dispersion coefficient $D_{T}^{M}$ of the motile bacteria (for the nonmotile ones, the effect is quite undetectable). The values of $D_{T}^{M}$ are represented in Fig. 5(b), and the linear regression of the data yields $D_{T}^{M}=$ $D_{0}^{M}+\zeta_{T}^{M} U$ with a dispersivity $\zeta_{T}^{M}=1.5 \pm 0.1 \mu \mathrm{m}$ much smaller than the longitudinal one. This transverse dispersion is yet another feature that distinguishes the transport of motile microorganisms from nonmotile ones.

The transport characteristics obtained for motile and nonmotile bacteria reveal that motility has two major effects that may seem contradictory and contraintuitive: (1) a retardation of the transport for a large number of motile bacteria and at the same time and (2) a rapid downstream progression of some others. To help understand these two antagonistic observations, we analyze in the following the statistical properties of the trajectories from a more microscopic point of view in order to identify some elementary mechanisms influencing the transport process. 

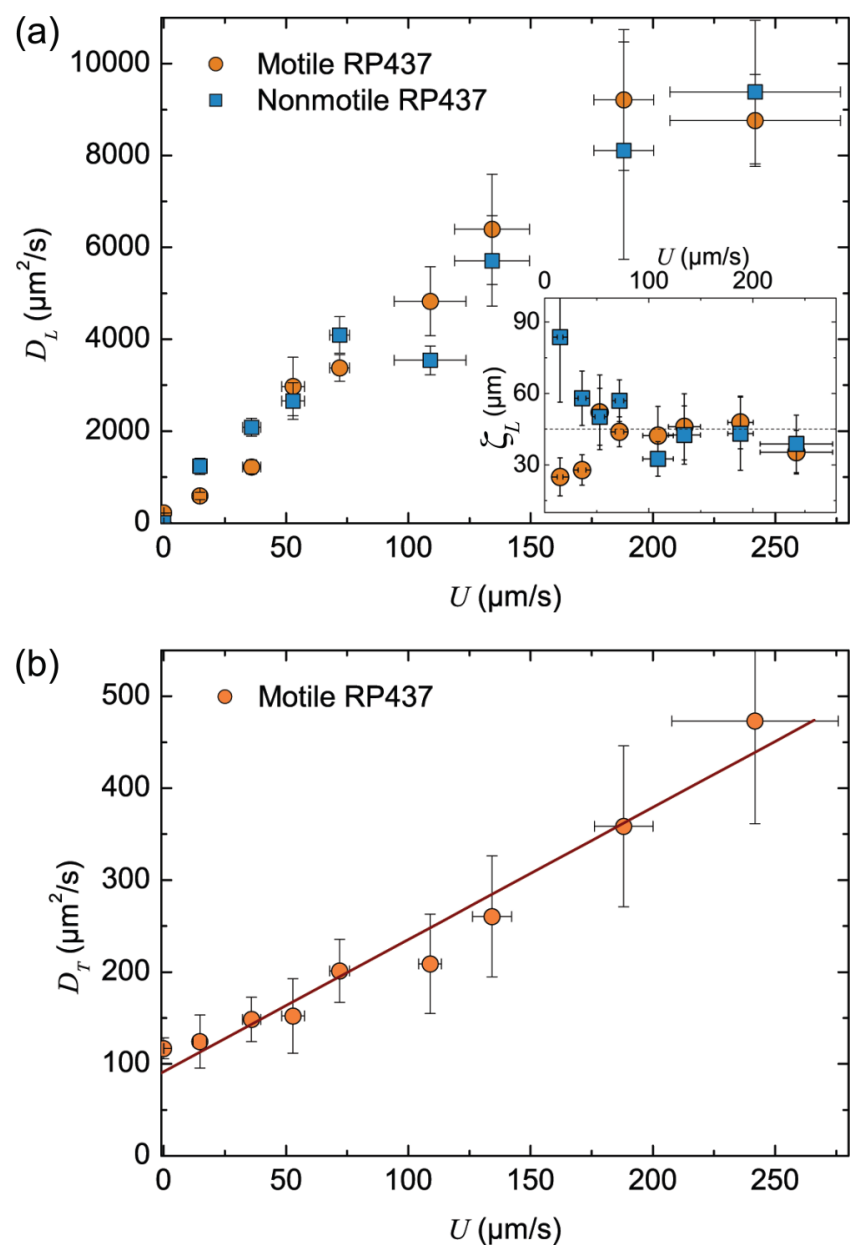

FIG. 5. (a) Longitudinal dispersion coefficient $D_{L}$ as a function of $U$ for motile and nonmotile bacteria. Inset: Dispersivity $\zeta_{L}^{N M}=\frac{D_{L}^{N M}}{U}$ and $\zeta_{L}^{M}=\frac{D_{L}^{M}-D_{0}^{M}}{U}$ as a function of $U$ for nonmotile and motile bacteria, respectively. The dotted line represents the constant function $y=50 \mu \mathrm{m}$. (b) Transverse dispersion coefficient $D_{T}$ of motile bacteria. The solid line represents a linear regression of the data yielding $\zeta_{T}^{M}=1.5 \pm 0.1 \mu \mathrm{m}$.

\section{Dynamical trapping}

\section{Staying in the grain vicinity}

First, we study the influence of the motility on the presence of bacteria in the vicinity of the pillar surfaces (the grains). The trajectories are divided into "fluid" and "grain" sections (see Fig. 6) characterizing the presence of the microorganisms in these domains.

The "fluid" sections correspond to subparts of the trajectories where the distance bacteria/ obstacle is larger than $\delta=1.5 \mu \mathrm{m}$ while the "grain" sections correspond to the parts of the trajectories for which the distance bacteria/obstacle is less than $1.5 \mu \mathrm{m}$. The average duration of the "fluid" segments, $\tau_{f}$, and "grain" segments, $\tau_{g}$, are computed and averaged over all the trajectories. The relative fractions of time, $P_{\text {surf }}=\frac{\tau_{g}}{\tau_{f}+\tau_{g}}$, for the motile bacteria and the nonmotile bacteria are shown in Fig. 7(a). One observes a striking difference between the motile and the nonmotile bacteria.

For nonmotile bacteria, the average flow velocity $U$ has no influence on $P_{\text {surf }}$, and its value remains significantly lower than the value of the motile ones. The relative amount of time spent 


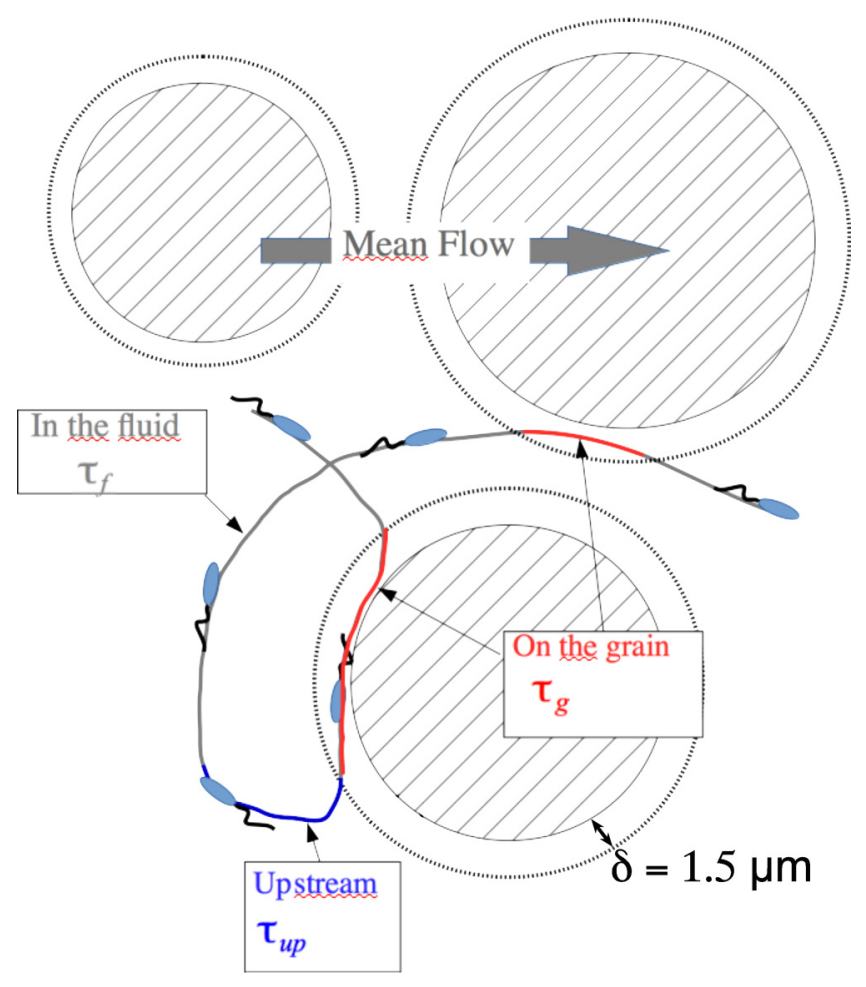

FIG. 6. Schematics of a bacterium trajectory and the corresponding parameters as defined in the text. $\tau_{\text {up }}$ is the period of time spent with a negative $v_{x}, \tau_{g}$ is the period of time spent on the grain vicinity, i.e., when the distance bacteria/obstacle is smaller than $\delta=1.5 \mu \mathrm{m}$, and $\tau_{f}$ is the period of time spent in the fluid when the distance bacteria/obstacle is larger than $\delta=1.5 \mu \mathrm{m}$.

around the obstacles is approximately four times greater for the motile bacteria than for the nonmotile ones, and this effect seems to be more pronounced when flow velocities become comparable to the bacteria velocity.

We also quantify $\kappa$, the frequency of encounters of the bacteria with the grain surfaces. Then we compute the number of contacts divided by the track duration and average this quantity over all tracks. In Fig. 7(b) we show the values of $\kappa$ for motile and nonmotile bacteria. For nonmotile bacteria this frequency remains constant over the whole range of fluid velocities but also remains significantly lower than its counterpart for motile bacteria. The ratio can be as large as 5 when the fluid velocities become comparable to the bacteria swimming velocity.

These sharp differences prove that motility promotes the flow of bacteria towards the obstacles and at the same time increases the residence time of the swimmers at the surfaces.

\section{Upstream motion}

Upon careful observation of movies recorded at high magnification (see the Supplemental Material at Ref. [41]) we see an impressive number of motile bacteria moving upstream (i.e., with a negative velocity in the laboratory reference frame), while this behavior seems absent for nonmotile bacteria. To quantify the upstream swimming, trajectories are segmented into periods during which the bacteria move either downstream or upstream. The average durations of these portions of trajectories, $\tau_{\text {up }}$ and $\tau_{\text {down }}$, are calculated. Figure 8 displays the relative time $P_{\text {up }}=\frac{\tau_{\text {up }}}{\tau_{\text {up }}+\tau_{\text {down }}}$ showing a clear difference between the values measured for motile and nonmotile bacteria: the periods during 

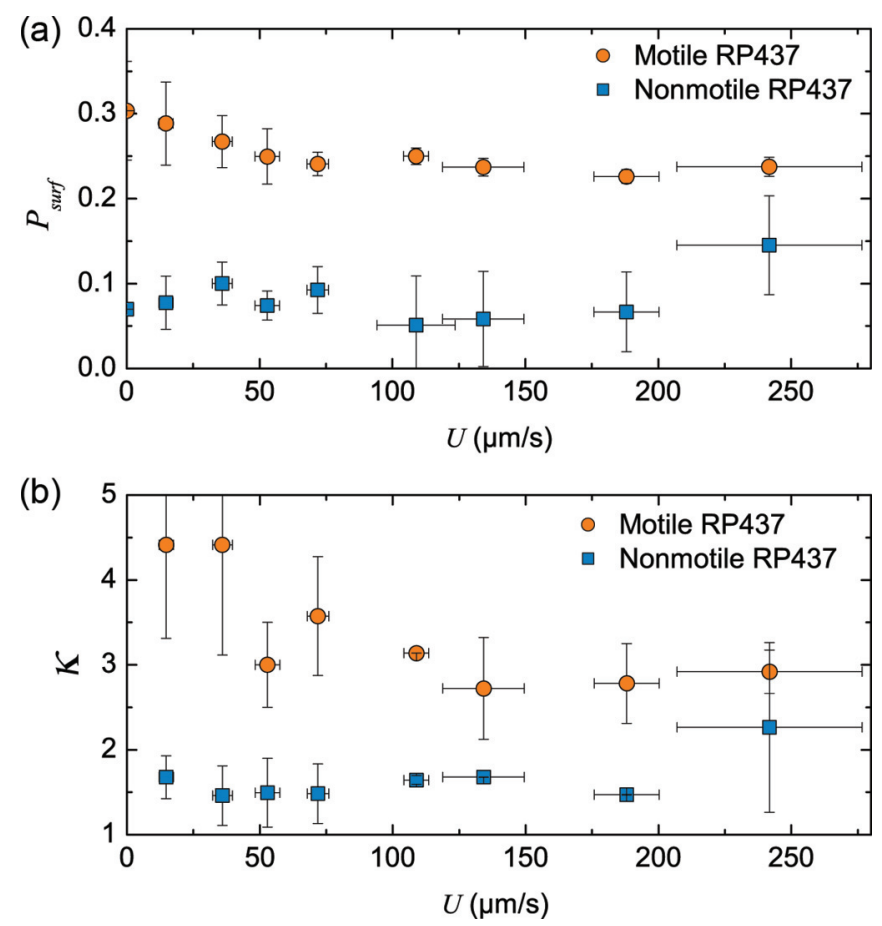

FIG. 7. (a) Average time spent by the bacteria swimming in the vicinity of an obstacle $\tau_{g}$ normalized by the total time $\tau_{g}+\tau_{f}$, where $\tau_{f}$ is the average time spent in the fluid as a function of $U$. (b) Mean number of occurrences a bacterium gets in contact with an obstacle per unit of time $\tau=d / U$.

which motile bacteria move upstream are very long, whereas for nonmotile ones the upstream motion is almost absent.

This analysis clearly demonstrates that motility favors the flow of bacteria toward regions of low velocity, most of the time close to the grains where they perform upstream motion. This is a dynamical trapping effect that will contribute to a global transfer of motile bacteria at an average velocity lower than the fluid velocity. This observation is a microscopic explanation of the retardation effect reported previously for the dispersion curves.

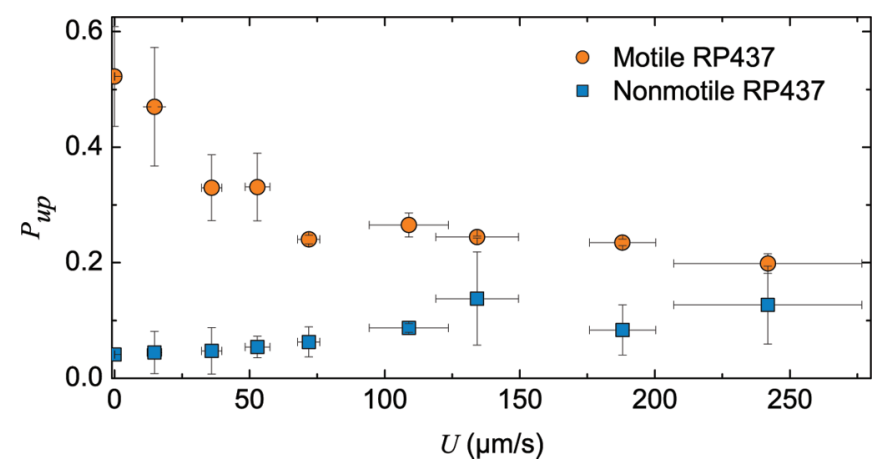

FIG. 8. Average duration of the upstream displacement $\tau_{\text {up }}$ normalized by the total time $\tau_{\text {up }}+\tau_{\text {down }}$, where $\tau_{\text {down }}$ is the average duration of the downstream $\left(v_{x}>0\right)$ displacement as a function of $U$. 


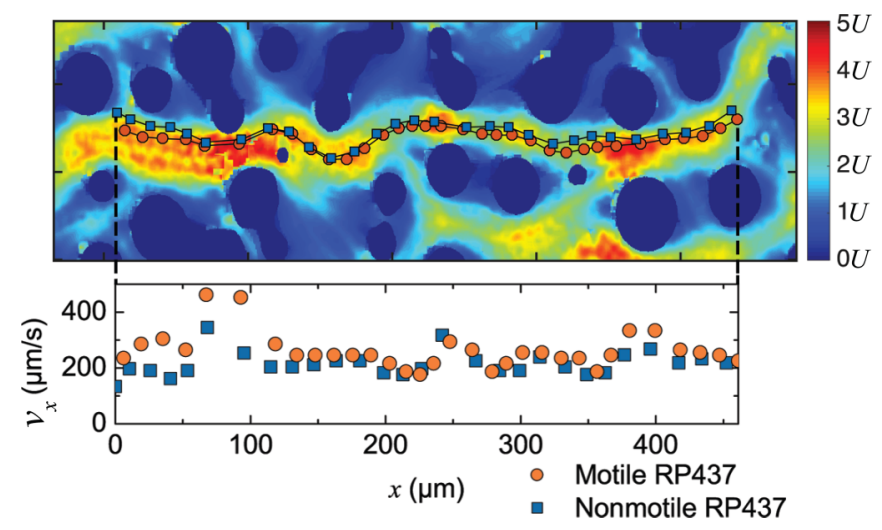

FIG. 9. Overlap of two close trajectories of a motile (orange circles) and a nonmotile (blue squares) bacteria with the local fluid velocity. The inset shows the speed of the bacteria $v_{x}$ as a function of the longitudinal position $x$. Experiments are performed at $U=72 \mu \mathrm{m} / \mathrm{s}$.

\section{E. Rapid downstream migration}

Let us come back now to the second observation concerning the rapid downstream progression of some motile bacteria. A close look at the velocity field reveals the existence of channels connecting the inlet and the outlet of the porous medium (within the visualization window) and along which the fluid velocity is at least twice the average fluid velocity $U$ (see Fig. 9). We called these channels the "fast tracks." The bacteria moving at a stream-wise velocity at least twice the mean flow velocity will be called fast (or slow otherwise). To illustrate this "fast-track" mechanism enhancing the transport of motile bacteria in the fastest channels, we overlay onto the velocity map two very close trajectories, one for a motile bacterium and the other for a nonmotile one (see Fig. 9).

In the inset, the velocities along the streamwise coordinate are displayed. We see in this example that the motile bacterium is always traveling faster than the nonmotile one. We also notice a systematic increase in the velocity difference as they cross the pore constrictions. The flow in these regions seems to align the bacteria along the flow direction, thus enhancing their mean transport velocity. Interestingly, the effect of stabilization by constriction was also described and discussed recently by Potomkin et al. [42].

In Fig. 10(a) we display the mean velocity of the fast bacteria $V_{\text {fast }}$ as a function of the mean flow $U$. Then it appears that the population of fast motile bacteria is transported at a velocity in average higher than the population of fast nonmotile ones. We anticipate that the fast bacteria are essentially transported along the "fast tracks."

To further quantify this effect, each trajectory is split into "fast" and "slow" sections. The relative duration of the "fast" segments $P_{\text {fast }}=\frac{\tau_{\text {fast }}}{\tau_{\text {fas }}+\tau_{\text {slow }}}$ are displayed in Fig. 10(b) for both motile and nonmotile bacteria and for all the flow velocities $U$ studied. It is immediately noted that, except for the lowest velocities, motile bacteria stay in the fast streams longer than the nonmotile bacteria.

\section{SUMMARY AND CONCLUSION}

To conclude, we have shown that microbial motility has a significant impact on the average transport and dispersion properties of bacterial suspensions flowing inside a porous medium.

By tracking swimming and nonswimming bacteria up to a transport scales of typically six times the pore size, we identify a phenomenology contrasting sharply between the motile and the nonmotile bacteria. For the nonmotile bacteria, one recovers the classical results of geometrical hydrodynamic dispersion characterizing the transport of inert species inside a porous medium $[37,38]$. This is essentially the phenomenology expected for dilute colloidal suspensions with no 

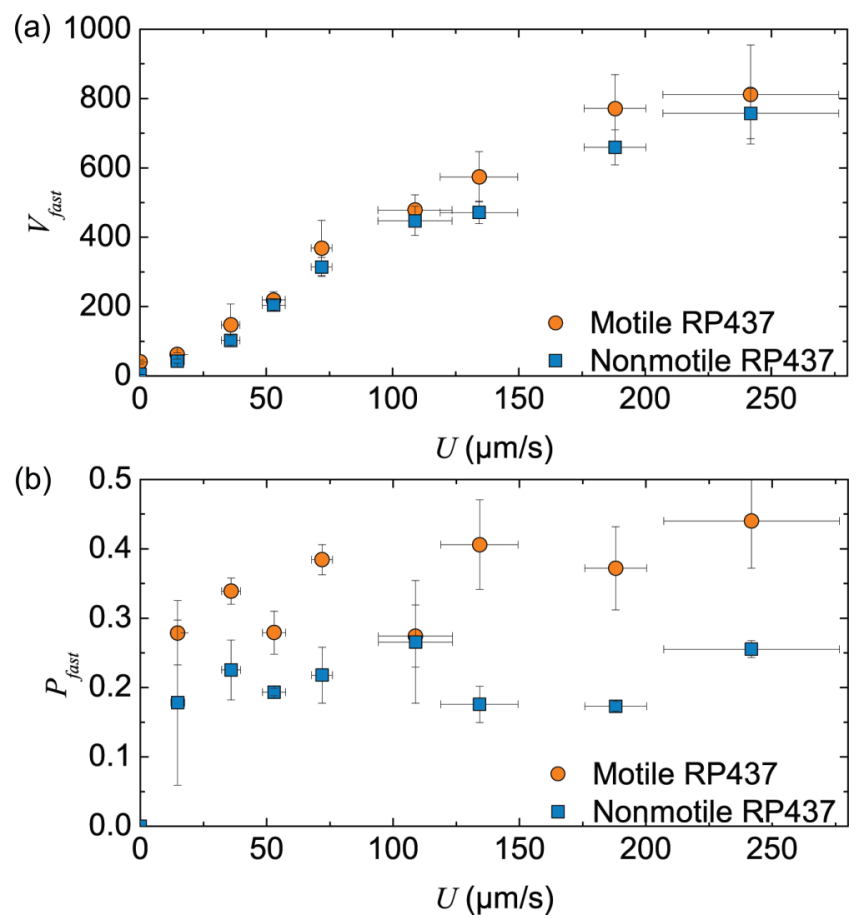

FIG. 10. (a) Speed of the fastest detected bacteria as a function of the mean flow velocity $U$. (b) Average duration, $\tau_{\text {fast }}$, of the sequences during which a bacteria is moving with a speed $v_{x}$ larger than twice the average flow velocity, normalized by $\tau_{\text {fast }}+\tau_{\text {slow }}$, where $\tau_{\text {slow }}$ is the averaged duration of the sequences during which $v_{x}<2 U$.

adhesion on solid surfaces, provided a particle size smaller than the pore throat. In this case the mean transport velocity is the mean flow velocity, and the longitudinal dispersion coefficient is proportional to the flow velocity, with a dispersivity length scale congruent to a pore size.

However, for the active species the ability to swim across the flow lines and eventually to dwell at surfaces or, opposite this, to take preferentially fast channels, changes drastically the distribution of bacteria in the flow. The shape of the mean distribution of longitudinal displacements at a given lag time is no longer a Gaussian distribution as for the nonmotile ones. In the streamwise direction, the mode of the distribution comes before the mean, hence characterizing a retardation effect due to the motile character of the bacteria. The curve is akin to a $\Gamma$ distribution characterized by a positive skewness and an upstream front decaying exponentially. Remarkably, this non-Gaussian distribution seems to hold for mean transport distances significantly larger than the pore size. For motile bacteria the mean transport velocity is systematically retarded with respect to the nonmotile ones. This "dynamical trapping" effect as we describe it does not result from any chemical or physical interaction often invoked to explain transport retardation, but essentially is borne in the swimming activity of the bacteria spending a significant time close to the surfaces and also swimming upstream. This is in stark contrast with the nonmotile species, which essentially follow the flow lines. Opposite this, we also measured a contribution that could explain the exponential forefront as the motile bacteria seem to take fast channels for a significantly long time and add up their swimming contribution to the maximal flows. Interestingly, this "fast-track" effect identified in the context of a model porous medium seems to be consistent with the observations of several laboratory-scale columns and field experiments which reported the presence of early breakthroughs of microorganisms [43-45]. Note that our dispersion measurements do not correspond to the predictions made by Alonso-Matilla et al. [18], as in their case the dispersion process remains close 
to a Taylor-dispersion mechanism. In our case, we have something closer to a geometrical dispersion process with a scaling for the diffusivity coefficient linear in the flow velocity $\left(D \sim P_{e}\right)$. Moreover, the retardation mechanism we observe displays a negative skewness, whereas for Alonso-Matilla et al. the retardation curve displays a positive one. Therefore from a theoretical standpoint, the issue of dispersion of active particles in random porous media remains a challenging and open issue.

In spite of these marked differences between motile and nonmotile species, the scaling of the mean longitudinal dispersion with the flow velocity are qualitatively similar (proportional to the mean flow). Importantly, the motile bacteria display transverse dispersion, an effect which is almost undetectable for the nonmotile one.

From these results emerges a new understanding on how the motility of many microorganisms influences in depth the transport processes and the spatial distribution in natural environments such as porous soils, fractured rocks, or even biological networks. We already foresee two important practical implications. First, for bacterial communities transported in a flow, the active retention effect leads to a thorough exploration of surfaces. This would influence strongly the chances for adhesion or biofilm formation. Second, because motility favors an efficient longitudinal transport for a subpopulation of swimmers, it will increase the volume explored by pioneering bacteria with important implications for the development of forefront contamination and colonization.

Remarkably, in the last years, active matter studies have brought to the forefront many paradigmatic shifts in the understanding of the out-of-equilibrium organization of motile bacterial suspensions [46]. In this context, standard notions such as the equations of state [47], phase transitions [48,49], Brownian or Fickean diffusion [50,51], or the rheological response [52-56] had to be deeply revisited to account for the crucial importance of motility. Consequently, transport, dispersion, or filtration of motile microorganisms in porous media is likely to yield results that differ qualitatively from those of passive colloids.

These effects are unique transport properties in addition to the many singular features already identified for active matter. But it is also interesting to realize that they impact positively the natural ability of bacterial populations to survive, grow, and reach their ecological niche.

\section{ACKNOWLEDGMENTS}

This work is supported by public grants overseen by the French National Research Agency (ANR), ANR Bacflow AAPG 2015, and from the Laboratoire d'Excellence Physics Atom Light Mater (LabEx PALM) as part of the Investissements d'Aveni program (reference: ANR-10LABX-0039). We acknowledge support by the Universidad de Buenos Aires (UBACyT No. 20020130100570BA) and the LIA PMF-FMF (Franco-Argentinian International Associated Laboratory in the Physics and Mechanics of Fluids). H.A. and A.C. thank D. Bouville for his help in the clean room.

[1] J. C. Anderson, E. J. Clarke, A. P. Arkin, and C. A. Voig, Environmentally controlled invasion of cancer cells by engineered bacteria, J. Mol. Biol. 355, 619 (2006).

[2] O. Felfoul et al., Magneto-aerotactic bacteria deliver drug-containing nanoliposomes to tumour hypoxic regions, Nat. Nanotechnol. 11, 941 (2016).

[3] L. R. Brown, Microbial enhanced oil recovery (MEOR), Curr. Opin. Microbiol. 13, 316 (2010).

[4] F.-G. Simon, T. Meggyes, and C. McDonald (eds.), Advanced Groundwater Remediation: Active and Passive Technologies (Thomas Telford, London, 2002).

[5] A. Karimi, S. Yazdi, and A. M. Ardekani, Hydrodynamic mechanisms of cell and particle trapping in microfluidics, Biomicrofluidics 7, 021501 (2013).

[6] T. R. Ginn et al., Processes in microbial transport in the natural subsurface, Adv. Water Resour. 25, 1017 (2002). 
[7] P. K. Pandey et al., Contamination of water resources by pathogenic bacteria, AMB Express 4, 51 (2014).

[8] M. Y. Corapcioglu and A. Haridas, Microbial transport in soils and groundwater: A numerical model, Adv. Water Resour. 8, 188 (1985).

[9] T. C. Peterson and R. C. Ward, Development of a bacterial transport model for coarse soils, J. Am. Water Resour. Assoc. 25, 349 (1989).

[10] M. J. Hendry, J. R. Lawrence, and P. Maloszewski, Effects of velocity on the transport of two bacteria through saturated sand, Ground Water 37, 103 (1999).

[11] J. W. A. Foppen, A. Mporokoso, and J. F. Schijven, Determining straining of Escherichia coli from breakthrough curves, J. Contam. Hydrol. 76, 191 (2005).

[12] N. Tufenkji, Modeling microbial transport in porous media: Traditional approaches and recent developments, Adv. Water Resour. 30, 1455 (2007).

[13] H. Bai et al., Comparison of transport between two bacteria in saturated porous media with distinct pore size distribution, RSC Adv. 6, 14602 (2016).

[14] G. Lutterodt, M. Basnet, J. W. A. Foppen, and S. Uhlenbrook, The effect of surface characteristics on the transport of multiple Escherichia coli isolates in large scale columns of quartz sand, Water Res. 43, 595 (2009).

[15] G. Lutterodt, J. W. A. Foppen, A. Maksoud, and S. Uhlenbrook, Transport of Escherichia coli in $25 \mathrm{~m}$ quartz sand columns, J. Contam. Hydrol. 119, 80 (2011).

[16] T. A. Camesano and B. E. Logan, Influence of fluid velocity and cell concentration on the transport of motile and nonmotile bacteria in porous media, Environ. Sci. Technol. 32, 1699 (1998).

[17] M. W. Becker et al., Bacterial transport experiments in fractured crystalline bedrock, Ground Water 41, 682 (2003).

[18] R. Alonso-Matilla, B. Chakrabarti, and D. Saintillan, Asymptotic transport and dispersion of active particles in periodic porous media, arXiv:1809.00086 [physics.flu-dyn].

[19] D. Takagi et al., Hydrodynamic capture of microswimmers into sphere-bound orbits, Soft Matter 10, 1784 (2014).

[20] S. Spagnolie et al., Geometric capture and escape of a microswimmer colliding with an obstacle, Soft Matter 11, 3396 (2015).

[21] L. Tao and R. M. Ford, Enhanced transverse migration of bacteria by chemotaxis in a porous T-sensor, Environ. Sci. Technol. 43, 1546 (2009).

[22] R. Rusconi, J. F. Guasto, and R. Stocker, Bacterial transport suppressed by fluid shear, Nat. Phys. 10, 212 (2014).

[23] Marcos, H. C. Fu, T. R. Powers, and R. Stocker, Bacterial rheotaxis, Proc. Natl. Acad. Sci. USA 109, 4780 (2012).

[24] P. D. Frymier, R. M. Ford, H. C. Berg, and P. T. Cummings, Three-dimensional tracking of motile bacteria near a solid planar surface, Proc. Natl. Acad. Sci. USA 92, 6195 (1995).

[25] J. Hill, O. Kalkanci, J. L. McMurry, and H. Koser, Hydrodynamic Surface Interactions Enable Escherichia coli to Seek Efficient Routes to Swim Upstream, Phys. Rev. Lett. 98, 068101 (2007).

[26] T. Kaya and H. Koser, Direct upstream motility in Escherichia coli, Biophys. J. 102, 1514 (2012).

[27] J. Kantsler, M. Dunkel, and R. E. Blayney, Goldstein, Rheotaxis facilitates upstream navigation of mammalian sperm cells, eLife 3, e02403 (2014).

[28] N. Figueroa-Morales et al., Living on the edge: Transfer and traffic of E. coli in a confined flow, Soft Matter 11, 6284 (2015).

[29] A. P. Berke, L. Turner, H. C. Berg, and E. Lauga, Hydrodynamic Attraction of Swimming Microorganisms by Surfaces, Phys. Rev. Lett. 101, 038102 (2008).

[30] G. Miño et al., E. coli accumulation behind an obstacle, Adv. Microbiol. 8, 451 (2018).

[31] E. Altshuler et al., Flow-controlled densification and anomalous dispersion of E. coli through a constriction, Soft Matter 9, 1864 (2013).

[32] S. D. Ryan, G. Ariel, and A. Be'er, Anomalous fluctuations in the orientation and velocity of swarming bacteria, Biophys. J. 111, 247 (2016).

[33] M. Sidortsov, Y. Morgenstern, and A. Be'er, Role of tumbling in bacterial swarming, Phys. Rev. E 96, 022407 (2017). 
[34] C. Douarche, A. Buguin, H. Salman, and A. Libchaber, E. Coli and Oxygen: A Motility Transition, Phys. Rev. Lett. 102, 198101 (2009).

[35] A. D Edelstein et al., Advanced methods of microscope control using $\mu$ Manager software, J. Biol. Methods 1, e10 (2014).

[36] Fiji freeware image analysis, https://imagej.net/Fiji/.

[37] P. G. Saffman, A theory of dispersion in a porous medium, J. Fluid Mech. 6, 321 (1959).

[38] J. Bear, Dynamics of Fluids in Porous Media (Elsevier, Amsterdam, 1971).

[39] G. E. Uhlenbeck and L. S. Ornstein, On the theory of the Brownian motion, Phys. Rev. 36, 823 (1930).

[40] M. Wu et al., Collective bacterial dynamics revealed using a three-dimensional population-scale defocused particle tracking technique, Appl. Environ. Microbiol. 72, 4987 (2006).

[41] See Supplemental Material at http://link.aps.org/supplemental/10.1103/PhysRevFluids.4.013102 for a video.

[42] M. Potomkin, A. Kaiser, L. Berlyand, and I. Aranson, Focusing of active particles in a converging flow, New J. Phys. 19, 115005 (2017).

[43] G. M. Hornberger, A. L. Mills, and J. S. Herman, Physical and chemical factors influencing transport of microorganisms through porous media, Water Resour. Res. 28, 915 (1992).

[44] D. R. McCaulou, R. C. Bales, and J. F. McCarthy, Use of short-pulse experiments to study bacteria transport through porous media, J. Contam. Hydrol. 15, 1 (1994).

[45] C. Stumpp, J. R. Lawrence, M. J. Hendry, and P. Maloszewski, Transport and bacterial interactions of three bacterial strains in saturated column experiments, Environ. Sci. Technol. 45, 2116 (2011).

[46] M. C. Marchetti et al., Hydrodynamics of soft active matter, Rev. Mod. Phys. 85, 1143 (2013).

[47] A. P. Solon et al., Pressure is not a state function for generic active fluids, Nat. Phys. 11, 673 (2015).

[48] F. Ginelli, F. Peruani, M. Bär, and H. Chaté, Large-Scale Collective Properties of Self-Propelled Rods, Phys. Rev. Lett. 104, 184502 (2010).

[49] G. Gregoire and H. Chaté, Onset of Collective and Cohesive Motion, Phys. Rev. Lett. 92, 025702 (2004).

[50] X.-L. Wu and A. Libchaber, Particle Diffusion in a Quasi-Two-Dimensional Bacterial Bath, Phys. Rev. Lett. 84, 3017 (2000).

[51] P. Galajda, J. Kleymer, P. Chaikin, and R. Austin, A wall of funnels concentrates swimming bacteria, J. Bacteriol. 189, 8704 (2007).

[52] Y. Hatwalne, S. Ramaswamy, M. Rao, and R. A. Simha, Rheology of Active-Particle Suspensions, Phys. Rev. Lett. 92, 118101 (2004).

[53] A. Sokolov and I. S. Aranson, Reduction of Viscosity in Suspension of Swimming Bacteria, Phys. Rev. Lett. 103, 148101 (2009).

[54] S. Rafaï, L. Jibuti, and P. Peyla, Effective Viscosity of Microswimmer Suspensions, Phys. Rev. Lett. 104, 098102 (2010).

[55] D. Saintillan, The dilute rheology of swimming suspensions: A simple kinetic model, Exp. Mech. 50, 1275 (2010).

[56] H. M. López, J. Gachelin, C. Douarche, H. Auradou, and E. Clément, Turning Bacteria Suspensions into Superfluids, Phys. Rev. Lett. 115, 028301 (2015). 